\title{
Indução floral de limeiras ácidas 'Tahiti’ submetidas a baixas temperaturas
}

\author{
Flower induction of acid lime trees 'Tahiti' subjected to low temperature
}

\author{
Dierlei dos Santos $^{\mathrm{I}}$ Dalmo Lopes de Siqueira $^{\mathrm{I}}$ Paulo Roberto Cecon ${ }^{\mathrm{II}}$
}

\section{RESUMO}

\begin{abstract}
Este trabalho foi realizado com o objetivo de quantificar a necessidade de acúmulo de horas de frio para a floração da limeira ácida 'Tahiti', sem a aplicação de deficiência hídrica, além de avaliar as trocas gasosas e fluorescência da clorofila a em condições de baixa temperatura e baixa luminosidade. O experimento foi conduzido em Viçosa$M G$, Brasil, com plantas de limeira ácida 'Tahiti', enxertadas sobre limoeiro 'Cravo', cultivadas em vasos de 12 litros. O delineamento utilizado foi o inteiramente casualizado, com sete tratamentos e quatro repetições, com uma planta por parcela. A partir do dia 01/02/2008 até o dia 30/04/2008, quatro plantas foram retiradas da câmara de crescimento (CC) (temperatura: $16 / 12^{\circ} \mathrm{C}$ (dia/noite); umidade relativa: $70 \%$; fotoperíodo: 10 horas; densidade de fluxo de fótons fotossintéticos: $100 \mu \mathrm{mol} \mathrm{m} \mathrm{m}^{-2} \mathrm{~s}^{-1}$ ) a cada 15 dias e levadas para a casa de vegetação $(C V)$ sem controle ambiental, totalizando sete datas de transferência. A exposição de plantas a baixas temperaturas, sob condições controladas não causou alterações significativas nas características de fluorescência da clorofila a, mas causou redução de $80 \%$ nas trocas gasosas. Entretanto, essa redução não prejudicou a floração das plantas. As plantas expostas a condições não indutoras apenas emitiram brotações vegetativas, enquanto aquelas submetidas a baixas temperaturas, quanto maior foi o tempo de exposição, maior foi o número de flores emitidas.
\end{abstract}

Palavras-chave: floração, Citrus latifolia Tanaka, ambiente controlado.

\section{ABSTRACT}

The research was realized to quantify the need for accumulation hours under low temperatures to the acid lime 'Tahiti' flowering, without water application and beyond evaluating gas exchange and chlorophyll a fluorescence under low temperature and light. The experiment was conducted in Viçosa-MG, Brazil, with plants from acid lime 'Tahiti' grafted on Rangpur lime grown in pots of 12 liters. The design was completely randomized with seven treatments and four replicates with one plant per plot. From 02/01/2008 until 04/30/2008, four plants were taken from each growth chamber (CC) (temperatures: $16 / 12^{\circ} \mathrm{C}$ (day/night); air humidity: $70 \%$; photoperiod: 10 hours; photosynthetic photon flux density: $100 \mu \mathrm{mol} \mathrm{m} \mathrm{m}^{-2} \mathrm{~s}^{-1}$ ) to 15 days and taken to a greenhouse (CV) without environmental control, totaling seven dates of transfer. Exposure of plants to low temperatures, under controlled conditions, does not cause significant changes in the characteristics of chlorophyll a fluorescence, but caused $80 \%$ of reduction in gas exchange. This reduction does not hurt the flowering plants. Plants exposed to no-induced conditions only issued vegetative shoots, while those submitted to lower temperatures, the greater was the exposure time, the greater was the number of flowers sent.

Key words: flowering, Citrus latifolia Tanaka, controlled environment.

\section{INTRODUÇÃO}

Temperaturas que oscilam entre 13 a $15^{\circ} \mathrm{C}$ durante o dia e 10 a $13^{\circ} \mathrm{C}$ à noite são consideradas promotoras da floração dos citros (MOSS, 1969). Apesar da existência de pesquisas procurando determinar as faixas ideais de temperatura necessárias para a indução floral, o limite máximo para que o processo ocorra não é conhecido, acreditando-se estar em torno de $19^{\circ} \mathrm{C}$.

'Departamento de Fitotecnia, Universidade Federal de Viçosa (UFV), 36570-000, Viçosa, MG, Brasil. E-mail: dierlei@vicosa.ufv.br. *Autor para correspondência.

IDepartamento de Estatística, UFV, Viçosa, MG, Brasil. 
Temperaturas acima de $22^{\circ} \mathrm{C}$ são consideradas ineficientes (DAVENPORT, 1990). ALBRIGOet al. (2002) afirmam que a floração ocorre quando há acúmulo de 800 a $1.000 \mathrm{~h}$ de temperaturas abaixo de $20^{\circ} \mathrm{C}$ sem interrupções no período e temperaturas máximas acima de $26,6^{\circ} \mathrm{C}$ e mínimas superiores a $21,1^{\circ} \mathrm{C}$ por mais de sete dias consecutivos, que são temperaturas que estimulam a emissão de novas brotações. Para KRAJEWSKI \& RABE (1995), o efeito das baixas temperaturas sobre a indução floral do citros não está demonstrado de forma definitiva, pois seu efeito, sobre o aumento no número de flores é seguido também por aumentos significativos nos números de brotações vegetativas.

A condição indutora ao florescimento é ao mesmo tempo estressante para a planta cítrica, portanto várias mudanças fisiológicas ocorrem nesse período, principalmente relacionadas à fotossíntese. Nos meses de inverno, período em que ocorre a indução floral natural, há queda na atividade fotossintética (MEDINA, 2002; RIBEIRO, 2006; RIBEIRO \& MACHADO, 2007; MACHADO, 2009). Essa queda na fotossíntese ocorre devido à queda na radiação solar, menor disponibilidade de água no solo, temperaturas diurnas/noturnas mais baixas e queda da demanda por fotoassimilados pela planta, devido ao menor crescimento. Qualquer tipo de estresse ou situação que reduza o consumo dos produtos fotoquímicos pela diminuição da eficiência de carboxilação poderá gerar excesso de energia de excitação(BAKER \& ROSENQVIST, 2004). Porém, em condições em que a fixação de $\mathrm{CO}_{2}$ é pequena, mas limitada pela baixa temperatura e densidade de fluxo de fótons fotossintéticos $(D F F F)$, situação comum em experimentos conduzidos em câmaras de crescimento, espera-se que não ocorra excesso de energia de excitação que necessite ser dissipada.

O trabalho foi realizado com o objetivo de quantificar a necessidade de acúmulo de horas de frio necessárias para a floração da limeira ácida 'Tahiti' sobre limoeiro 'Cravo', sem a presença de deficiência hídrica, além de avaliar as trocas gasosas e fluorescência da clorofila $a$ em condições de baixa temperatura e baixa luminosidade.

\section{MATERIAL E MÉTODOS}

O experimento foi conduzido no período de fevereiro a abril de 2008 em câmara de crescimento (CC) e em casa de vegetação (CV), no setor de Fruticultura, localizado no Campus da Universidade Federal de Viçosa. Foram utilizadas plantas de limeira ácida 'Tahiti' (Citrus latifolia Tanaka) com três anos de idade, enxertadas sobre limoeiro 'Cravo' (Citrus limonia
Osbeck), cultivadas em vasos de 12 litros em CV sob condições ambientais não controladas.

Em meados de janeiro, quando as condições ambientais não são indutoras ao florescimento na região, as plantas foram transferidas para $\mathrm{CC}$, ajustada para operar com temperatura de $25 / 20^{\circ} \mathrm{C}$ (dia/noite), umidade relativa de $70 \%$, fotoperíodo de 15 horas, DFFF de $170 \mu \mathrm{mol} \mathrm{m}^{-2} \mathrm{~s}^{-1}$ para adaptação das plantas. No início de fevereiro, a CC foi regulada para operar com temperatura de $16 / 12^{\circ} \mathrm{C}$ (dia/noite), umidade relativa de $70 \%$, fotoperíodo de 10 horas, DFFF de $100 \mu \mathrm{mol} \mathrm{m}^{-2} \mathrm{~s}^{-1}$. No dia desse ajuste, o primeiro grupo de plantas foi removido da CC e levado para a CV. Nessa fase, as plantas foram irrigadas a cada dois dias para evitar que o estresse hídrico fosse um fator de interferência.

O experimento foi conduzido em delineamento inteiramente casualizado, com sete tratamentos e quatro repetições, com uma planta por parcela. A partir do dia 01/02/2008 até o dia 30/04/2008, quatro plantas foram retiradas da $\mathrm{CC}$ a cada 15 dias e levadas para a CV sem controle ambiental, cuja temperatura média situou-se por volta $\operatorname{dos} 25^{\circ} \mathrm{C}$, totalizando sete datas de transferência, que foram consideradas como tratamentos. Portanto, as plantas foram submetidas a $0,360,720,1080,1440,1800$ e 2160 horas de frio, correspondendo a $0,15,30,45,60,75$ e 90 dias de permanência na CC.

Durante o período experimental, as quatro primeiras plantas transferidas (testemunha) para a CV, e outras quatro das que permaneceram na $\mathrm{CC}$, foram submetidas a avaliações quinzenais dos parâmetros de fluorescência da clorofila $a$ e trocas gasosas nas folhas. A fluorescência foi medida usando-se um fluorômetro ADC FIM 1500 (ADC Bioscientific Ltd, Hodderston, UK) (fluorescência direta). Foram avaliadas a fluorescência inicial $\left(F_{o}\right)$, a fluorescência máxima $\left(F_{m}\right)$ e a eficiência fotoquímica máxima do FSII $\left(F_{v} / F_{m}\right)$. As leituras foram realizadas pela manhã, utilizando-se sempre a quarta folha madura a partir do ápice, de ramos localizados na região mediana e exposta da planta.

A taxa de assimilação líquida do carbono $(A)$, a condutância estomática $\left(g_{s}\right)$ e a transpiração $(E)$ foram avaliadas quinzenalmente e também pela manhã, em folhas semelhantes às descritas anteriormente. Foi utilizado um analisador de gases a infravermelho (IRGA - LCpro+, Analytical Development Company, Hoddesdon, Reino Unido), em sistema aberto, sob um regime de luz de $100 \mu \mathrm{mol} \mathrm{m}^{-2} \mathrm{~s}^{-1}$, temperatura na câmara de análise de $16^{\circ} \mathrm{C}$ e concentração ambiente de $\mathrm{CO}_{2}$ para leituras realizadas no interior da $\mathrm{CC}$, e um regime de luz de $1300 \mu \mathrm{mol} \mathrm{m}^{-2} \mathrm{~s}^{-1}$, temperatura na câmara de 
análise de $25^{\circ} \mathrm{C}$ e concentração ambiente de $\mathrm{CO}_{2}$ quando as leituras foram realizadas em CV.

Após a transferência das plantas para a CV, foram feitas irrigações diárias. As plantas de todos os tratamentos iniciaram a brotação cerca de 20 dias após a transferência para a CV. Nas brotações, foram avaliados o número de brotos vegetativos e número de flores emitidas. Os brotos emitidos foram classificados em vegetativos (presença só de folhas), floríferos multiflorais (presença de mais de uma flor, sem folhas), floríferos uniflorais (presença de uma única flor, sem folhas), mistos multiflorais (presença de flores e folhas) e mistos uniflorais (presença de uma flor e folhas). Os resultados foram expressos em porcentagem (número de brotos e flores por 100 nós).

Os dados da floração foram analisados por meio da análise de variância. Os modelos, ajustados por meio de regressão, foram escolhidos com base na significância dos coeficientes de regressão no nível de $5 \%$ de probabilidade pelo teste " $t$ ", de Student, no coeficiente de determinação e no potencial para explicar o fenômeno biológico. Para as características fluorescência da clorofila $a$ e trocas gasosas, utilizouse a estatística descritiva.

\section{RESULTADOS E DISCUSSÃO}

A manutenção das plantas em CC não causou alterações significativas nas características de fluorescência da clorofila $a$ quando comparadas às plantas mantidas em CV (Figuras 1A, B e C). Observouse, de modo geral, que $F_{o}$ e $F_{\sqrt{ }} / F_{m}$ foram levemente superiores nas plantas mantidas em CC, mas não diferentes da $\mathrm{CV}$. A característica $F_{m}$ foi a única que realmente foi diferente nos dois ambientes, sendo maior em plantas mantidas em CC. O aumento em $F_{m}$ possivelmente está associado à baixa $D F F F$ na CC, pois, em presença de alta irradiância, normalmente $\mathrm{a}_{m}$ é menor. Há energização da membrana dos tilacóides, motivada pelo aumento do gradiente de $\mathrm{pH}$ transtilacoidal e pela extinção não fotoquímica de energia de excitação, resultando na redução de $F_{m}$ (KRAUSE \& WEIS, 1991). Reduções nos valores de $F_{m}$ geralmente levam a reduções em $F_{v} / F_{m}$ (MACHADO, 2009). $F_{\sqrt{ }} / F_{m}$ representa a eficiência máxima que a luz absorvida pela antena do FSII é convertida em energia química (em condições adequadas, apresenta valores em torno de 0,80 ) e é um indicador da ocorrência de fotoinibição, quando plantas estão sujeitas a vários tipos de estresses do ambiente, incluindo o frio (BAKER \& ROSENQVIST, 2004 ). Quando a disponibilidade de energia de excitação é superior à capacidade de utilização na fotossíntese (reações bioquímicas), há fotoinibição, indicada por valores de $F_{v} / F_{m}$ menores que 0,725 (CRITCHLEY, 1998), valores não atingidos nas plantas nos dois ambientes. Em tecidos sadios, o aumento de $D F F F$ determina reduções em $F_{v} / F_{m}$, desde que exista dependência da fixação de $\mathrm{CO}_{2}$ em relação à eficiência fotoquímica e vice-e-versa (SCHREIBER \& BILGER, 1987). MACHADO (2009) verificou redução nos valores de $F_{\sqrt{ }} / F_{m}$ em laranjeiras 'Valência' submetidas ao frio noturno e concluiu que foi devido à fotoinibição. Possivelmente, essa redução não foi observada em plantas mantidas em CC, porque a fotossíntese, além de estar limitada pela baixa temperatura, também estava por baixa $D F F F$, ou seja, não havia excesso de energia que necessitasse ser dissipado.

Em condições de radiação solar natural, as plantas apresentaram trocas gasosas semelhantes às relatadas na literatura para citros (Figuras 1D, E e F), ou seja, $A$ em torno de $12 \mu \mathrm{mol} \mathrm{m} \mathrm{m}^{-2} \mathrm{~s}^{-1}, g_{s}$ próximo a $0,2 \mathrm{~mol} \mathrm{~m}^{-2} \mathrm{~s}^{-1} \mathrm{e} E$ em torno de $3,0 \mathrm{mmol} \mathrm{m}^{-2} \mathrm{~s}^{-1}$ (MEDINA, 2002; HABERMANN et al., 2003; RIBEIRO et al., 2004; RIBEIRO, 2006; RIBEIRO \& MACHADO, 2007; MACHADO, 2009; MAGALHÃES FILHO, 2009). Observou-se também que ocorreram poucas variações nessas características durante o período experimental. Uma possível justificativa é que o experimento foi conduzido em uma única estação do ano, havendo, portanto, poucas alterações nas condições climáticas (temperatura, umidade relativa e radiação solar). Por outro lado, as trocas gasosas das plantas mantidas em CC sofreram redução de cerca de $80 \%$, quando comparadas com aquelas mantidas em CV (Figuras 1D, E e F). Esse declínio na capacidade fotossintética pode ser devido à baixa $D F F F\left(100 \mu \mathrm{mol} \mathrm{m}^{-2} \mathrm{~s}^{-1}\right)$ e à baixa temperatura.

Sabe-se que a taxa máxima de fotossíntese em citros ocorre em temperaturas do ar em torno de $22^{\circ} \mathrm{C}$ a $25^{\circ} \mathrm{C}$ (RIBEIRO et al., 2004; MACHADO et al., 2005) e $D F F F$ entre $600-800 \mu \mathrm{mol} \mathrm{m}^{-2} \mathrm{~s}^{-1}$ (HABERMANN et al., 2003; MACHADO et al., 2002; RIBEIRO et al., 2003). MACHADO et al. (2005) encontraram, em estudo com limeira ácida 'Tahiti', que a taxa de assimilação de $\mathrm{CO}_{2}$ variou de $12 \mu \mathrm{mol} \mathrm{m}^{-2} \mathrm{~s}^{-1}$ para $3 \mu \mathrm{mol} \mathrm{m}^{-2} \mathrm{~s}^{-1}$, quando o $D F F F$ foi reduzido de 1000 para $100 \mu \mathrm{mol} \mathrm{m}^{-2} \mathrm{~s}^{-1}$, exatamente o que ocorreu nas condições desse ensaio. Em condições naturais, durante o inverno, a menor taxa de fotossíntese pode estar relacionada tanto com a menor demanda por fotoassimilados, devido à menor taxa de crescimento, quanto com a diminuição da temperatura do ar, do solo e do comprimento do dia (MACHADO et al., 2002; 2005; RIBEIRO \& MACHADO, 2007; RIBEIRO et al., 2009a, b). 


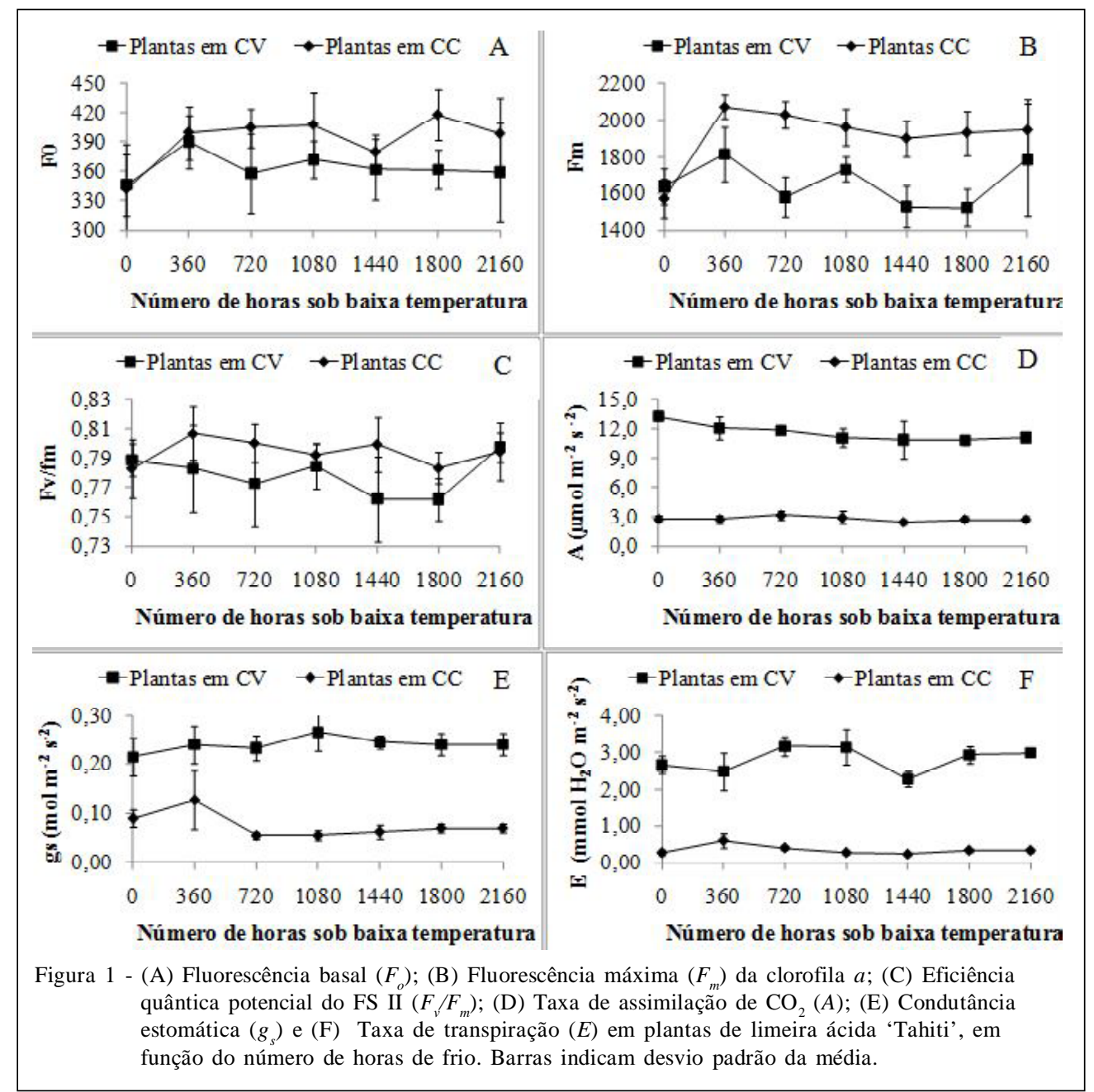

Os decréscimos acentuados de $g_{s}$ nas plantas submetidas ao resfriamento indicam que o fechamento dos estômatos é parcialmente responsável pelo decréscimo de $A$ (MACHADO, 2009). O fechamento parcial dos estômatos pode ser devido à inibição direta do frio sobre a fotossíntese diminuindo-a, o que causaria o aumento da concentração intercelular de $\mathrm{CO}_{2}$ (Ci) (ALLEN et al., 2000). Alternativamente, o estômato poderia ser o alvo direto do resfriamento, causando o abaixamento de $\mathrm{Ci}$ (ALLEN \& ORT, 2001; MACHADO et al., 2002: MEDINA, 2002). Esse fechamento estomático possivelmente foi o responsável também pela menor transpiração, pois ANGELOCCI et al. (2004) relacionaram menores valores de transpiração em limeira ácida 'Tahiti' durante o inverno, com o fechamento parcial dos estômatos, devido a baixas temperaturas.

As plantas que permaneceram na $\mathrm{CV}$, não submetidas a condições indutoras de florescimento, emitiram o maior número de brotações vegetativas. Por outro lado, para as plantas mantidas na $\mathrm{CC}$, quanto maior foi o acúmulo de baixas temperaturas, menor foi a emissão dessas brotações, chegando a ser nula quando as plantas permaneceram o maior período na CC (Figura 2A). Esses resultados comprovam que há uma relação quantitativa entre intensidade de floração e baixas temperaturas (SOUTHWICK \& DAVENPORT, 1986).

De modo inverso, quanto maior foi o tempo de exposição a baixas temperaturas, maior o número de flores emitidas, sendo nulo em plantas mantidas em CV (Figura 2B). As inflorescências do tipo multifloral predominaram, principalmente em plantas que permaneceram mais de 1080 horas sob baixas temperaturas (Figura 2C). Flores produzidas em inflorescências do tipo mista multifloral também apresentaram comportamento semelhante à anterior, porém com menor número de flores emitidas (Figura 2E).

A presença desses tipos de inflorescência é importante, pois as inflorescências com folhas 


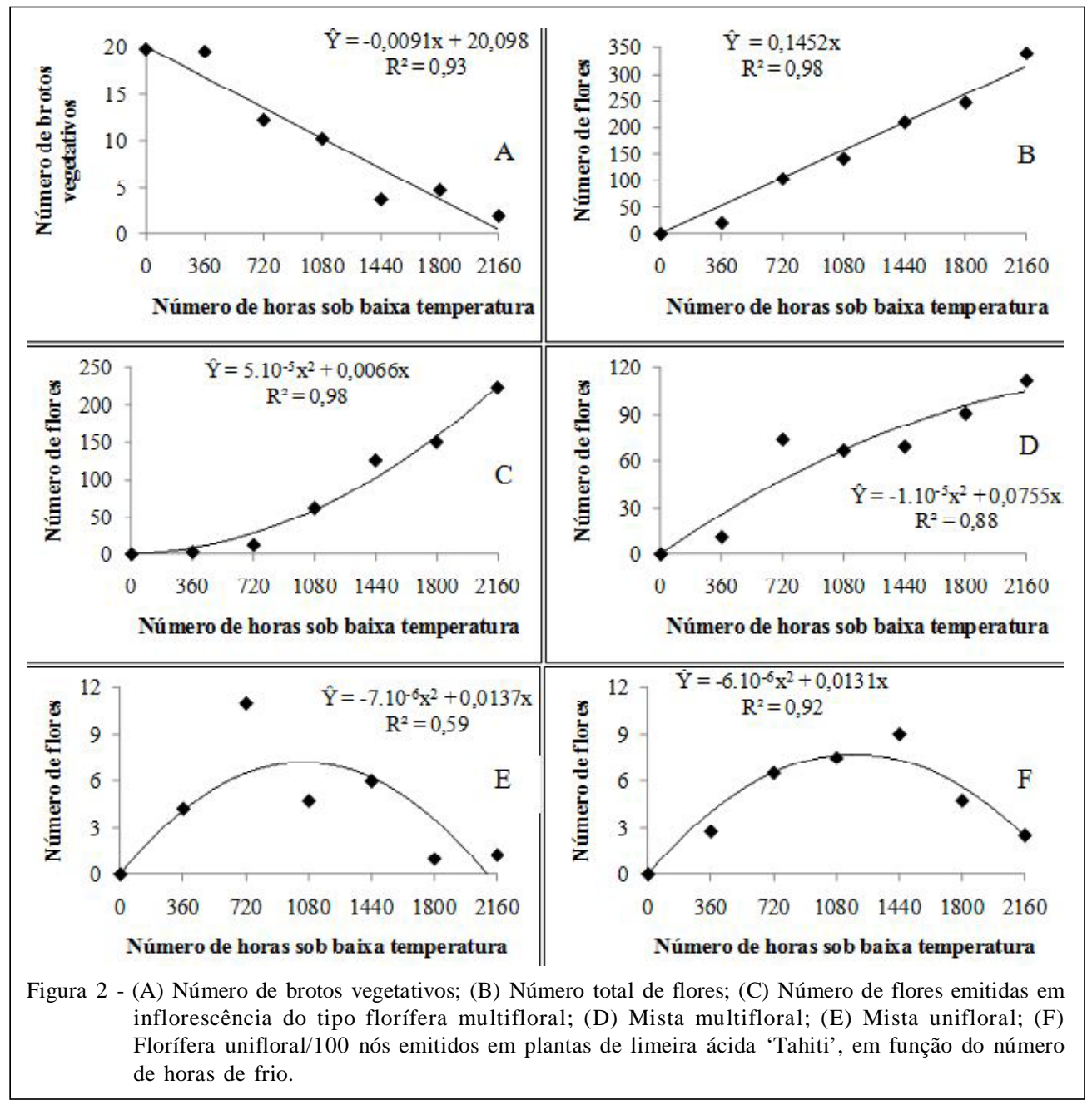

produzem relativamente mais frutos do que inflorescências sem folhas. Na inflorescência com folhas, o fruto cresce mais rapidamente e apresenta maior probabilidade de permanecer na planta até a colheita, em relação aos frutos de inflorescências sem folhas (RUIZ et al., 2001). Frutos produzidos em inflorescências com folhas têm pegamento entre quatro a seis vezes maior, em relação aos produzidos em inflorescências que não têm folhas. A contribuição das folhas deve-se, provavelmente, a fatores hormonais e nutricionais (SAIDHA et al., 1985).

LELIS et al. (2008) relataram que plantas de tangerineira 'Ponkan', que não foram expostas às baixas temperaturas, praticamente não produziram flores, mesmo expostas por longos períodos à temperatura favorável à brotação, e que, para as condições climáticas de Viçosa-MG, o estresse hídrico não foi necessário para a floração. Essa observação pode ser estendida também para a limeira ácida 'Tahiti', o que permite afirmar que os citros florescem apenas com indução por frio, dispensando o efeito do estresse hídrico, que, nas condições climáticas da maior parte do Sudeste brasileiro, ocorre simultaneamente ao período de frio. É possível que a influência promotora do déficit hídrico e da baixa temperatura sobre o florescimento seja na supressão do crescimento vegetativo (SPIEGEL-ROY \& GOLDSCHMIDT, 1996), portanto a presença de um ou outro é suficiente para que ocorra a floração.

ALBRIGO et al. (2002) estabeleceram três diferentes níveis de indução de floração para citros. Pesquisa realizada por LELIS et al. (2008) com 'Ponkan' confirmou essa classificação, bem como os resultados obtidos neste trabalho. ALBRIGO et al. (2002) consideraram que o nível de indução é baixo quando o acúmulo de horas abaixo de $20^{\circ} \mathrm{C}$ é menor que 720 . Nessa condição, o estímulo não é suficiente para induzir a uma floração significativa, que resulte em produção econômica. O nível médio de indução ocorre quando o número de horas abaixo de $20^{\circ} \mathrm{C}$ varia entre 750 e 1200 
horas. Nesse caso, pode ocorrer indução suficiente para garantir uma produção econômica. Por último, o nível alto, que ocorre quando o número de horas de acúmulo de temperaturas abaixo de $20^{\circ} \mathrm{C}$ está acima de 1200 horas, sendo o estímulo suficiente para induzir a uma boa produção.

Foi pequeno o número de flores produzidas em inflorescências uniflorais e mistas uniflorais. Essas características apresentaram comportamento quadrático com o aumento do acúmulo das horas de frio, sendo que, em ambos os casos, o número máximo de flores emitidas ocorreu quando o acúmulo foi de 1080 horas de frio (Figuras 2E e F). Flores produzidas nessas inflorescências são mais sensíveis a estresses e sofrem abscisão com mais facilidade, quando comparadas às flores produzidas nos demais tipos de inflorescências.

As plantas mantidas em CC, mesmo com as taxas de trocas gasosas reduzidas, limitadas pela luz e temperatura, mantiveram o metabolismo suficiente para manter o florescimento satisfatório, principalmente naquelas que permaneceram maior período de tempo sob baixa temperatura. Essa redução é semelhante ao que acontece sob condições naturais, pois, nos meses de baixa temperatura e baixa disponibilidade hídrica (inverno), ocorre redução da fotossíntese e paralisação aparente do crescimento (MACHADO et al., 2002). Apesar da queda na assimilação de $\mathrm{CO}_{2}$, há acúmulo de reservas nas folhas, suficiente para prover a demanda do florescimento (RIBEIRO, 2006; PRADO et al., 2007).

\section{CONCLUSÃO}

A exposição de plantas a baixas temperaturas, sob condições controladas, não causou alterações significativas nas características de fluorescência da clorofila $a$, mas causou redução de $80 \%$ nas trocas gasosas. Entretanto, essa redução não prejudicou a floração das plantas. As plantas expostas a condições não indutoras apenas emitiram brotações vegetativas, enquanto aquelas expostas a baixas temperaturas, quanto maior foi o tempo de exposição, maior foi o número de flores emitidas.

\section{REFERÊNCIAS}

ALBRIGO, L.G. et al. Flowering expert system development for a phenology based citrus decision support system. Acta Horticultural, v.584, p.247-254, 2002.

ALLEN, D.J.; ORT, D.R. Impacts of chilling temperatures on photosynthesis in warm-climate plants. Trends in Plant Science, v.6, p.36-42, 2001.
ALLEN, D.J. et al. An overnight chill induces a delayed inhibition of photosynthesis at midday in mango (Mangifera indica L.). Journal of Experimental Botany, v.51, p.18931902, 2000.

ANGELOCCI, L.R. et al. Transpiration, leaf diffusive conductance, and atmospheric water demand relationship in an irrigated acid lime orchard. Brazilian Journal of Plant Physiology, v.16, p.53-64, 2004. Disponível em: <http:// www.scielo.br/pdf/bjpp/v16n1/a08v16n1.pdf>. Acesso em: 15 ago. 2010. doi: 10.1590/S1677-04202004000100008.

BAKER, N.R.; ROSENQVST, E. Applications of chlorophyll fluorescence can improve crop production strategies: an examination of future possibilities. Journal of Experimental Botany, v.55, p.1607-1621, 2004. Disponível em: <http:// jxb.oxfordjournals.org/cgi/reprint/55/403/1607>. Acesso em: 15 ago. 2010. doi:10.1093/jxb/erh196.

CRITCHLEY, C. Photoinhibition. In: RAGHAVENDRA, A.S. Photosynthesis: a comprehensive treatise. Cambridge: Cambridge University, 1998. Cap.20, p.264-272.

DAVENPORT, T.L. Citrus flowering. Horticultural Reviews, v.12, p.349-408, 1990.

HABERMANN, G. et al. Gas exchange rates at different vapor pressure deficits and water relations of 'Pera' sweet orange plants with citrus variegated chlorosis. Scientia Horticulturae, v.98, p.233-245, 2003.

KRAJEWSKI, A.J.; RABE, E. Citrus flowering: a critical evaluation. Journal of Horticultural Science, v.70, p.357374, 1995.

KRAUSE, G.H.; WEIS, E. Chlorophyll fluorescence and photosynthesis: the basics. Annual Review of Plant Physiology and Plant Molecular Biology, v.42, p.313-349, 1991.

LELIS, F.M.V. et al. Florescimento de tangerineiras 'Ponkan' (Citrus reticulata Blanco) submetidas a diferentes períodos de temperatura invernal. Revista Brasileira de Fruticultura, v.30, p.818-821, 2008. Disponível em: <http://www.scielo.br/ pdf/rbf/v30n3/43.pdf>. Acesso em: 20 ago. 2010. doi: 10.1590/ S0100-29452008000300043.

MACHADO, D.F.S.P. A resposta da fotossíntese à baixa temperatura noturna em laranjeira 'Valência' é dependente do porta-enxerto. 2009. 58f. Dissertação (Mestrado em Tecnologia da Produção Agrícola) - Instituto Agronômico de Campinas, SP.

MACHADO, E.C. et al. Respostas da fotossíntese a fatores ambientais em três espécies de citros. Pesquisa Agropecuária Brasileira, v.40, p.1161-1170, 2005. Disponível em: <www.scielo.br/pdf/pab/v40n12/27505.pdf>. Acesso em: 20 ago. 2010. doi: 10.1590/S0100-204X2005001200002.

MACHADO, E.C. et al. Variação sazonal da fotossíntese, condutância estomática e potencial da água na folha de laranjeira 'Valência'. Scientia Agricola, v.59, p.53-58, 2002. Disponível em: 〈http://www.scielo.br/pdf/sa/v59n1/8073.pdf〉. 
Acesso em: 25 ago. 2010. doi: 10.1590/S010390162002000100007 .

MAGALHÃES FILHO, J.R. Resposta da fotossíntese à variação da temperatura radicular em laranjeira 'valência' enxertada em limoeiro 'cravo'. 2009. $50 \mathrm{f}$. Dissertação (Mestrado em Tecnologia da Produção Agrícola) Instituto Agronômico de Campinas, SP.

MEDINA, C.L. Fotossíntese, relações hídricas e alterações bioquímicas em laranjeira 'Pêra' com CVC e submetida à deficiência hídrica. 2002. 147f. Tese (Doutorado em Biologia Vegetal) - Instituto de Biologia, Universidade Estadual de Campinas, SP.

MOSS, G.I. Influence of temperature and photoperiod on flower induction and inflorescence development in sweet orange (Citrus sinensis L. Osbeck). Journal of Horticultural Science, v.44, p.311-320, 1969.

PRADO, A.K.S. et al. Florescimento e frutificação em laranjeiras 'Valência' com diferentes cargas de frutos e submetidas ou não à irrigação. Bragantia, v.66, p.173-182, 2007. Disponível em: <http://www.scielo.br/pdf/brag/v66n2/01.pdf>. Acesso em: 20 ago. 2010. doi: 10.1590/S000687052007000200001 .

RIBEIRO, R.V. et al. Growth- and leaf-temperature effects on photosynthesis of sweet orange seedlings infected with Xylella fastidiosa. Plant Pathology, v.53, p.334-340, 2004. Disponível em: <http://onlinelibrary.wiley.com/doi/10.1111/ j.0032-0862.2004.01012.x/pdf >. Acesso em: 20 ago. 2010. doi: $10.1046 / \mathrm{j} .1365-3059.2004 .01012 . x$.

RIBEIRO, R.V. et al. Early photosynthetic responses of sweet orange plants infected with Xylella fastidiosa. Physiological and Molecular Plant Pathology, v.62, p.167-173, 2003.

RIBEIRO, R.V.; MACHADO, E.C. Some aspects of citrus ecophysiology in subtropical climates: re-visiting photosynthesis under natural conditions. Brazilian Journal of Plant Physiology, v.19, p.393-411, 2007. Disponível em: <http://www.scielo.br/pdf/bjpp/v19n4/a09v19n4.pdf>. Acesso em: 30 ago. 2010. doi: 10.1590/S1677-04202007000400009.
RIBEIRO, R.V. et al. Photosynthesis and water relations of well-watered orange plants as affected by winter and summer conditions. Photosynthetica, v.47, p.215-222, 2009. Disponível em: <http://www.springerlink.com/content/ 1q844876680w85k0/fulltext.pdf>. Acesso em: 28 ago. 2010. doi: 10.1007/s11099-009-0035-2.

RIBEIRO, R.V et al. Seasonal and diurnal changes in photosynthetic limitation of young sweet orange trees. Environmental and Experimental Botany, v.66, p.203211, 2009. Disponível em: <http://www.sciencedirect.com/ science?_ob=MImg\&_imagekey=B6T66-4VY2C96-1 $7 \&$ \& c d i $=5022 \&$ _ u s e r $=687369 \&_{\text {_ p i i }=S}$ $\begin{array}{lllllllllllllllllllllllll}0 & 0 & 9 & 8 & 8 & 4 & 7 & 2 & 0 & 9 & 0 & 0 & 0 & 6 & 5 & 3 & \&\end{array}$ se a r c h \& c o ver Dat e = $05 \% 2$ F $31 \% 2$ F 200 $9 \&$ \& $k=999339997 \&$ view $=\mathrm{c} \& \mathrm{wchp}=\mathrm{dGLbVzW}-$ zSkzV\&md5=7b75c709a1dd7c1011e967ab7023a1 ea\&ie=/ sdarticle.pdf>. Acesso em: 28 ago. 2010. doi: 10.1016/ j.envexpbot.2009.03.011.

RIBEIRO, R.V. Variação sazonal da fotossíntese e relações hídricas da laranjeira 'Valência'. 2006. 157f. Tese (Doutorado em Física do Ambiente Agrícola) - Escola Superior de Agricultura "Luiz de Queiroz", SP.

RUIZ, A. et al. Carbohydrate availability in relation to fruitlet abscission in Citrus. Annals of Botany, v.87, p.805-812, 2001. Disponível em: <http://aob.oxfordjournals.org/cgi/ reprint/87/6/805>. Acesso em: 28 ago. 2010. doi: 10.1006/ anbo. 2001.1415 .

SAIDHA, T. et al. Endogenous cytokinins from developing "Shamouti" orange fruits derived from leafy and leafless inflorescences. Horticultural Science, v.26, p.35-41, 1985.

SCHREIBER, U.; BILGER, W. Rapid assessment of stress effects on plant leaves by chlorophyll fluorescence measurements. In: TENHUNEN, J.D. et al. Plant response to stress. Berlin: Springer Verlag, 1987. p.27-53. (NATO ASI Series G: Ecological Sciences, 15).

SOUTHWICK, J.M.; DAVENPORT, T.L. Characterization of water stress and low temperature effects on flower induction in citrus. Plant Physiology, v.81, p.26-29, 1986.

SPIEGEL-ROY, P.; GOLDSCHMI DT, E.E. Biology of citrus. Cambridge: Cambridge University, 1996. 230p. 